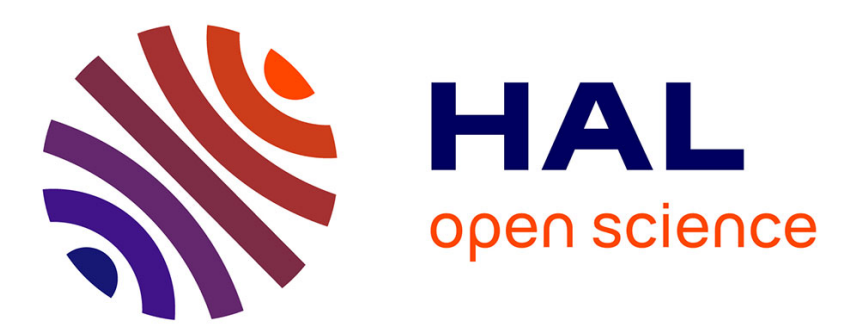

\title{
Growth and relaxation of a ridge on a soft poroelastic substrate
}

\author{
Menghua Zhao, François Lequeux, Tetsuharu Narita, Matthieu Roché, \\ Laurent Limat, Julien Dervaux
}

\section{- To cite this version:}

Menghua Zhao, François Lequeux, Tetsuharu Narita, Matthieu Roché, Laurent Limat, et al.. Growth and relaxation of a ridge on a soft poroelastic substrate. Soft Matter, 2018, 14, pp.61-72. hal01570135

\section{HAL Id: hal-01570135 \\ https://hal.science/hal-01570135}

Submitted on 28 Jul 2017

HAL is a multi-disciplinary open access archive for the deposit and dissemination of scientific research documents, whether they are published or not. The documents may come from teaching and research institutions in France or abroad, or from public or private research centers.
L'archive ouverte pluridisciplinaire HAL, est destinée au dépôt et à la diffusion de documents scientifiques de niveau recherche, publiés ou non, émanant des établissements d'enseignement et de recherche français ou étrangers, des laboratoires publics ou privés. 


\title{
Growth and relaxation of a ridge on a soft poroelastic substrate
}

\author{
Menghua Zhao, ${ }^{1,2}$ François Lequeux, ${ }^{2}$ Tetsuharu Narita, ${ }^{2}$ \\ Matthieu Roché, ${ }^{1}$ Laurent Limat, ${ }^{1}$ and Julien Dervaux ${ }^{1, *}$ \\ ${ }^{1}$ Matière et Systèmes Complexes, CNRS UMR 7057, \\ Université Paris Diderot, SPC University, \\ 10 Rue A. Domon et L. Duquet, F-75013 Paris, France \\ ${ }^{2}$ Sciences et Ingénierie de la Matière Molle, CNRS UMR 7615, \\ École Supérieure de Physique et de Chimie Industrielles de la Ville de Paris (ESPCI) ParisTech, \\ PSL University, 10, Rue Vauquelin, F-75231 Paris Cedex 05, France
}

(Dated: July 28, 2017)

\footnotetext{
*E-mail address: julien.dervaux @ univ-paris-diderot.fr
} 
Elastocapillarity describes the deformations of soft materials by surface tensions. Although the vast majority of elastocapillarity experiments are performed on soft gels, because of their tunable mechanical properties, the theoretical interpretation of these data has been so far undertaken solely within the framework of linear elasticity, neglecting the porous nature of gels. We investigate in this work the deformation of a thick poroelastic layer with surface tension subjected to an arbitrary distribution of time-dependent axisymmetric surface forces. Following the derivation of a general analytical solution, we then focus on the specific problem of a liquid drop sitting on a soft poroelastic substrate. We investigate how the deformation and the solvent concentration field evolve in time for various droplet sizes. In particular, we show that the ridge height beneath the triple line grows logarithmically in time as the liquid migrates toward the ridge. We then study the relaxation of the ridge following the removal of the drop and show that the drop leaves long-lived footprints after removal that may affect surface and wetting properties of gel layers but also the motion of living cells on soft materials.

\section{INTRODUCTION}

Thanks to their tunable mechanical and physico-chemical properties, synthetic hydrogels and elastomers are involved in a broad range of applications, from uses in cell culture to control the differentiation and the migration of cells, to dew collection ${ }^{1}$ and the handling of fluids. ${ }^{2-4}$ Gelslike structures are also a hallmark of many living tissues. In many cases of practical interest as well as in a broad range of natural systems, gels are so compliant that minute forces, such as surface tension forces at interfaces, are strong enough to significantly deform those gels. Elastocapillary phenomena, involving a competition between bulk elasticity and surface energies, have been studied since the 60's and the pioneering work of Lester and Shanahan. ${ }^{5-10}$ However, thanks to advanced methods in elastomer synthesis and imaging techniques that have brought new detailed observations of gel deformations at interfaces, elastocapillarity has recently attracted a lot of attention in the scientific community. ${ }^{11-18}$ In particular, studies on the wetting of soft materials by sessile or moving droplets have uncovered new unexpected physical phenomena. ${ }^{19-23}$ Following these new observations, several studies have been devoted to the theoretical interpretation of these new experimental data ${ }^{24,25}$ and elastowetting phenomena are becoming increasingly well understood although several issues of fundamental importance remain open such as the selection of the 
contact angle or the effect of different surface tensions for the dry and wet part of the gel surface.

Despite these important advances, and while many experimental studies are performed on gels, most theoretical studies on elastowetting have assumed that the soft deformable substrates are purely (linear) elastic materials. It was only recently recognized that even some simple features of static elastowetting, such as the formation of the ridge beneath the triple line below a sessile drop, ${ }^{21}$ are in fact time dependent processes. This non-instantaneous response differs from that of a purely elastic solid, but the rate-limiting mechanism could be either network rearrangement (viscoelasticity), solvent diffusion (poroelasticity) or a combination of both. However, intriguing results, such as the coexistence of multiple phases at the contact line in recent indentations experiments, ${ }^{22}$ unambiguously highlights the need to use a multiphase model (such as the poroelastic theory) to rationalize these observations.

In the simplest case, a gel can undergo two modes of deformations. ${ }^{26}$ On a very short timescale following the sudden application of a force on a gel sample, the solvent molecules do not have time to diffuse and the gel behaves as an incompressible elastic solid while a hydrostatic pressure builds up within the liquid phase. ${ }^{26,27}$ On this short timescale, the gel can change its shape but not its volume. In reaction to this pressure however, the solvent molecules migrate. This long-range migration process occurs on timescales that depend on the size of the sample and allows the gel to change both its shape and its volume. ${ }^{28-31}$ Therefore, while incompressible on a short timescale, a gel is highly compressible on a long timescale. In the limit of small deformations, this behavior is well described by the theory of linear poroelasticity, initially developed by Biot in $1941^{32}$ to describe soil consolidation.

In general, however, not all crosslinks in polymer networks are permanent and part of them may be capable of dynamic dissociation and re-association, such as physical gels ${ }^{33}$ or interpenetrating gel networks. ${ }^{34,35}$ These reversible crosslinks, together with the rearrangement of the polymer chains and the viscosity of the solvent itself, endow the gels with additional viscoelastic properties. ${ }^{36-38}$ Because poro- and visco-elastic processes occur simultaneously in gels, their time-dependent mechanical response is in general rather complex ${ }^{39-43}$ and must be described by a poro-visco-elastic theory. Because of this interplay, and although several models of poro-viscoelasticity have been developed ${ }^{29,44-47}$, experimental protocols allowing the extraction of the poroand visco-elastic material parameters have been devised only recently. ${ }^{40,41}$ While some work has been done to incorporate the viscoelastic response of the gel within the theoretical framework ${ }^{23,48}$ of elastowetting, neither the poro-elastic response nor the poro-visco-elastic response has been 
incorporated so far within the theoretical framework that describes the time-dependent behavior of soft solids near the triple line.

In order to grasp the new physical effects induced by the poroelasticy of the substrate on elastowetting phenomena, we will neglect visco-elastic behaviors in the present work and we will study the time dependent behavior of a thick poroelastic substrate subjected to an arbitrary, but axisymmetric, time-dependent distribution of normal surface forces. In the next section, we briefly recall the field and constitutive equations of linear poroelasticity. Next, we present a general analytical solution to the poroelastowetting problem. We then investigate the specific case of the poroelastic deformation due to the deposition (and subsequent removal) of a hemispherical drop at the surface of the gel. We then discuss our findings and highlight future development as well as outstanding questions of broad scientific interest.

\section{MATHEMATICAL FORMULATION}

\section{A. Field and constitutive equations}

In the reference state, the poroelastic substrate is not subjected to any mechanical load, the initial concentration of solvent in the gel is homogeneous and given by $c_{0}$ while the chemical potential is $\mu_{0}$. In the deformed state, the system is described by the solvent concentration $c$, chemical potential $\mu$ and displacement field $\vec{u}$. In response to the application of an external force, the solvent is not in diffusive equilibrium anymore and evolves according to the constraint of the conservation of the number of solvent molecules:

$$
\frac{\partial c}{\partial t}+\vec{\nabla} \cdot \vec{J}=0
$$

where $\vec{J}$ is the flux of the solvent in the gel and is driven by spatial differences of the chemical potential. For simplicity, we will assume that the flux $\vec{J}$ of small molecules is given by Darcy's law:

$$
\vec{J}=\left(\frac{k}{\eta \Omega^{2}}\right) \vec{\nabla} \mu
$$

where $k$ is the permeability, $\eta$ is the viscosity of the solvent and $\Omega$ is the molar volume of the solvent. If the poroelastic substrate is a gel, note that the mobility $k /\left(\eta \Omega^{2}\right)$ can also be expressed in term of the swelling ratio $\lambda_{0}$ in the freely swollen state as $D /\left(\mathscr{N}_{a} \Omega k_{B} T\right)\left(\lambda_{0}^{3}-1\right) / \lambda_{0}^{3}$ where 
$\mathscr{N}_{a}$ is the Avogadro number and $D$ is the intrinsic diffusivity of the solvent molecules. The strain tensor $\varepsilon$ is defined as:

$$
\varepsilon=\frac{1}{2}\left(\vec{\nabla} \vec{u}+(\vec{\nabla} \vec{u})^{t}\right)
$$

In the framework of linear poroelasticity, the stress tensor $\sigma$ is given by:

$$
\sigma=2 G\left(\varepsilon+\frac{v}{1-2 v} \operatorname{Tr}(\varepsilon) I\right)-\frac{\mu-\mu_{0}}{\Omega} I
$$

where $G$ is the shear modulus, $v$ is the the Poisson ratio that characterizes the ability of a gel to absorb its solvent and $I$ is the identity tensor. We assume that solvent and polymer molecules are incompressible and consequently the local volume variation is given by the local variation of the solvent concentration. This molecular incompressibility condition reads:

$$
\operatorname{Tr}(\varepsilon)=\left(c-c_{0}\right) \Omega
$$

The mechanical equilibrium in the bulk of the poroelastic layer is described by the Navier equations:

$$
\vec{\nabla} \cdot=\overrightarrow{0}
$$

Combining the equations above we get:

$$
\frac{\partial c}{\partial t}=D^{\star} \triangle c
$$

where

$$
D^{\star}=\frac{2(1-v) G k}{(1-2 v) \eta}
$$

is an effective diffusion coefficient (also called the cooperative diffusion coefficient) and $\triangle$ is the Laplace operator. Note that the material parameters $G, k$ and thus $D^{\star}$ are effective parameters that depend on the initial state of the gel. In a nonlinear theory, they are also functions on the local deformation of the gel. Within the framework of linear poroelasticity however, we will assume that the deformed state is close enough to the initial state such that $G, k$ and $D^{\star}$ can be treated as constant material parameters. Finally, combining (3)-(4)-(5)-(6), we get: 


$$
G \Omega\left(\triangle \vec{u}+\frac{\Omega}{1-2 v} \vec{\nabla}\left(c-c_{0}\right)\right)=\vec{\nabla} \mu
$$

\section{B. Boundary conditions}

At the free boundary, the gel is subjected to a force distribution $\vec{t}$ and surface tension $\gamma_{s}$

$$
\sigma \cdot \vec{n}=\vec{f}+\gamma_{s} \vec{n}(\vec{\nabla} \cdot \vec{n})
$$

where $\vec{n}$ and $\vec{f}$ are the unit normal vector to the surface and traction forces exerted at the substrate boundary, respectively. $\gamma_{s}$ is the surface tension of the solid. We are mostly interested by the case of an impermeable gel and thus

$$
\left.\frac{\partial \mu}{\partial z}\right|_{z=0}=0
$$

In addition, we assume that the substrate is infinitely thick and thus the displacement and stress fields vanish for $z \rightarrow-\infty$

\section{DEFORMATION OF A POROELASTIC HALF-SPACE WITH SURFACE TENSION}

As mentioned in the introduction, we are interested in the response of a thick poroelastic substrate following the sudden application of a distribution of surface forces. For the sake of simplicity, and motivated by the specific question of elastowetting, we will focus here on axisymmetric force distribution only. With this choice of geometry, it is convenient to use cylindrico-polar coordinates $(r, z)$ and restrict ourselves to time-dependent axisymmetric fields for the solvent concentration $c(r, z, t)$, chemical potential $\mu(r, z, t)$ and displacement field $\vec{u}(r, z, t)$ :

$$
\vec{u}(r, z, t)=u(r, z, t) \vec{e}_{r}+v(r, z, t) \vec{e}_{z}
$$

Furthermore, we only consider in this paper the case where the traction force at the free boundary is purely normal, i.e $\vec{f} \propto \vec{e}_{z}$. Although we will consider later time-dependent forcing, we first focus on the step response of the system i.e when the surface force distribution is suddenly applied at $t=0$ and subsequently maintained for $t \geq 0$. We may therefore write $\vec{f}=f_{z}(r) H(t) \vec{e}_{z}$, where $H(t)$ is the Heaviside step function. Thus, we have at the free surface: 


$$
\begin{aligned}
& \sigma_{z z}(r, z=0, t)=f_{z}(r) H(t)+\frac{\gamma_{s}}{r} \frac{\partial}{\partial r}\left(r \frac{\partial v}{\partial r}\right) \\
& \sigma_{r z}(r, z=0, t)=0
\end{aligned}
$$

\section{A. Hankel transform}

In order to solve the equilibrium equations presented above, it is convenient to introduce the following Hankel transforms:

$$
\begin{aligned}
& u(r, z, t)=\int_{0}^{\infty} s \hat{u}(s, z, t) J_{1}(s r) \mathrm{d} s \\
& v(r, z, t)=\int_{0}^{\infty} s \hat{v}(s, z, t) J_{0}(s r) \mathrm{d} s \\
& c(r, z, t)=\int_{0}^{\infty} s \hat{c}(s, z, t) J_{0}(s r) \mathrm{d} s \\
& \mu(r, z, t)=\int_{0}^{\infty} s \hat{\mu}(s, z, t) J_{0}(s r) \mathrm{d} s
\end{aligned}
$$

where $J_{0}(z)$ and $J_{1}(z)$ are the zero and first order Bessel function of the first kind, respectively.

\section{B. Instantaneous deformation}

When a traction force $\vec{f}=f_{z}(r) H(t) \vec{e}_{z}$ is suddenly applied at the free surface at $t=0$, the liquid has no time to migrate and system first deforms instantaneously as an incompressible solid. This elastic deformation causes the chemical potential to drop below its equilibrium value $\mu_{0}$ and sets the fluid into motion. Using a superscript ${ }^{i}$ to denote the instantaneous response, the instantaneous concentration field $c^{i}$ is thus $c^{i}=c_{0}$. As a consequence of the molecular incompressibility constraint (5) the instantaneous deformation field $\vec{u}^{i}$ satisfies $\vec{\nabla} \cdot \vec{u}^{i}=0$ and thus $\triangle \mu^{i}=0$. From these two relations, it follows that each component of the displacement field $u^{i}$ and $v^{i}$ satisfies the biharmonic equation, i.e: $\triangle^{2} u^{i}=\triangle^{2} v^{i}=0$. The two fields are not independent and $u^{i}$ can be expressed in term of $v^{i}$ using $\vec{\nabla} \cdot \overrightarrow{u^{i}}=0$. Inserting the Hankel transforms into the biharmonic equation, we get a linear fourth-order ordinary differential equations for, say, $\hat{v}^{i}$ :

$$
\frac{\partial^{4} \hat{v}^{i}}{\partial z^{4}}-2 s^{2} \frac{\partial^{2} \hat{v}^{i}}{\partial z^{2}}+s^{4} \hat{v}^{i}=0
$$


The above ordinary differential equation is readily solved and involves four unknown coefficients (that depend on $s$ ). Two of them are cancelled to satisfy $\lim _{z \rightarrow-\infty} \hat{v}^{i}=0$. The two remaining functions are found by making use of the two boundary conditions (13)-(14) and we find:

$$
\hat{v}^{i}=\frac{\hat{f}_{z}(s)}{2 G} \frac{e^{s z}(1-s z)}{s\left(1+s \ell_{s}\right)}
$$

where $\ell_{s}=\gamma_{s} /(2 G)$ is the elastocapillary length and $\hat{f}_{z}(s)$ is the Hankel transform of the surface traction $f_{z}(r)$. The radial component $\hat{u}^{i}$ of the instantaneous displacement field is given by:

$$
\hat{u}^{i}=\frac{\hat{f}_{z}(s)}{2 G} \frac{e^{s z} z}{\left(1+s \ell_{s}\right)}
$$

\section{Final state}

On a long-enough time scale, the system eventually reaches a thermodynamic equilibrium in which the solvent flux vanishes $(\vec{J}=\overrightarrow{0})$ and the chemical potential relaxes to its equilibrium value $\mu_{0}$ (provided that the surface force vanishes for $r \rightarrow \infty$ ). In this final stationnary state (where quantities are denoted by a superscript ${ }^{f}$ ), $c^{f}$ does not depend on time anymore and the concentration field therefore satisfies $\triangle c^{f}=0$. Because the concentration field is Laplacian and $\mu^{f}=\mu_{0}$, the final displacement field $\vec{u}^{f}$ again satisfies a biharmonic equation $\triangle^{2} u^{f}=\triangle^{2} v^{f}=0$ which again leads to an equation of the form (19) and which solution is now:

$$
\hat{v}^{f}=\frac{\hat{f}_{z}(s)}{2 G} \frac{e^{s z}(2-2 v-s z)}{s\left(1+s \ell_{s}(2-2 v)\right)}
$$

\section{Time-dependent deformation}

We now turn to the resolution of the time dependent problem. Although the time dependence only appears explicitly in the diffusion equation (7), it cannot be solve independently because the boundary conditions involve $c, \mu$ and the displacement field $\vec{u}$. Using equations (3)-(4)-(5)-(6), as well as the boundary conditions (13) and $\lim _{z \rightarrow-\infty} \hat{v}=0$, the fields $\hat{c}, \hat{v}$ and $\hat{\mu}$ can be expressed in term of $\hat{u}$ : 


$$
\begin{array}{r}
\hat{c}=\hat{c}_{0}+\frac{1}{\Omega}\left(s \hat{u}+\frac{\partial \hat{v}}{\partial z}\right) \\
\hat{\mu}=\hat{\mu}_{0}+\frac{G \Omega}{s(1-2 v)}\left(2 s^{2}(1-v) \hat{u}+s \frac{\partial \hat{v}}{\partial z}+(1-2 v) \frac{\partial^{2} \hat{u}}{\partial z^{2}}\right) \\
\hat{v}=e^{s z} g(s, t)-\frac{1}{s} \frac{\partial \hat{u}}{\partial z}
\end{array}
$$

where the function $g(s, t)$ is defined as:

$$
g(s, t)=\frac{1}{G s+s^{2} \gamma_{s}}\left(\hat{f}_{z}(s)+\left.2 G s \hat{u}\right|_{z=0}+\left.s \gamma_{s} \frac{\partial \hat{u}}{\partial z}\right|_{z=0}\right)
$$

Plugging the above results in (7), we obtain the following non homogeneous fourth-order linear partial differential equation for $\hat{u}$ :

$$
\frac{\partial}{\partial t}\left(\frac{\partial^{2} \hat{u}}{\partial z^{2}}-s^{2} \hat{u}\right)=D^{\star}\left(\frac{\partial^{4} \hat{u}}{\partial z^{4}}-2 s^{2} \frac{\partial^{2} \hat{u}}{\partial z^{2}}+s^{4} \hat{u}\right)+s^{2} \frac{\partial g}{\partial z}
$$

This equation is supplemented by the two boundary conditions (14) and (11) at the free surface while $\lim _{z \rightarrow-\infty} \hat{u}=0$. Furthermore, the initial condition is given by $\hat{u}(s, z, 0)=\hat{u}^{i}$ where $\hat{u}^{i}$ is defined by (21). Because we are interested in a first time by the step response of the system , this problem is best solved by introducing the Laplace transform $\hat{U}(s, z, \omega)$ of $\hat{u}(s, z, t)$ defined as:

$$
\hat{U}(s, z, \omega)=\int_{0}^{\infty} e^{-\omega t} \hat{u}(s, z, t) \mathrm{d} t
$$

Plugging the above expression into the evolution equation (27) for $\hat{u}(s, z, t)$ and making use of the initial condition $\hat{u}(s, z, 0)=\hat{u}^{i}$, we now obtain a non homogeneous fourth order linear ordinary differential equation that is easily solved by using the four boundary conditions mentioned previously.

\section{E. Solution for a step forcing}

The full solution for $\hat{U}(s, z, \omega)$ is given in the appendix and we only write here the more compact result for the (Hankel-Laplace transform of the) deflection $\hat{Z}(s, \omega)=\hat{U}(s, z=0, \omega)$ of the free surface:

$$
\hat{Z}(s, \omega)=\frac{\hat{f}_{z}(s)}{2 G s} \frac{1}{\omega\left(1+s \ell_{s}\right)+s^{2} D^{\star} \frac{1-2 v}{1-v}\left(\frac{s \sqrt{D^{\star}}}{\sqrt{s^{2} D^{\star}+\omega}}-1\right)}
$$


where we have used the result that the Laplace transform of the Heaviside step function $H(t)$ is $1 / \omega$.The inverse Hankel-Laplace transform can be obtained by the use of the inverse Hankel transform together with the help of the Bromwich integral that gives the inverse Laplace transform:

$$
\zeta(r, t)=\frac{1}{2 \pi i} \int_{\chi-i \infty}^{\chi+i \infty} \mathrm{d} \omega \int_{0}^{\infty} \mathrm{d} s e^{\omega t} \hat{Z}(s, \omega) s J_{0}(s r)
$$

where $\chi$ is a real number such that the axis of integration $] \chi-i \infty, \chi+i \infty[$, which is parallel to the imaginary axis, lies at the right of the pole of the integrand in (30), ensuring that the contour of integration is in the region of convergence. This solution is the step response of a poroelastic half-space subject to an arbitrary radially-symetric distribution of normal surface forces applied at $t=0$ i.e $\vec{f}=f_{z}(r) H(t) \vec{e}_{z}$. Let us first note that the two limiting cases described previously can be recovered using the initial and final value theorems:

$$
\begin{array}{r}
\hat{\zeta}^{i}(s)=\lim _{t \rightarrow 0} \hat{\zeta}(s, t)=\lim _{\omega \rightarrow \infty} \omega \hat{Z}(s, \omega)=\frac{\hat{f}_{z}(s)}{2 G} \frac{1}{s\left(1+s \ell_{s}\right)} \\
\hat{\zeta}^{f}(s)=\lim _{t \rightarrow \infty} \hat{\zeta}(s, t)=\lim _{\omega \rightarrow 0} \omega \hat{Z}(s, \omega)=\frac{\hat{f}_{z}(s)}{2 G} \frac{(2-2 v)}{s\left(1+s \ell_{s}(2-2 v)\right)}
\end{array}
$$

The response of the gel is thus that of an incompressible solid at short time while it behaves as a compressible solid at long time. This apparent compressibility is due to the ability of the solvent to migrate and is quantified by the poroelastic Poisson ratio $v$. When $v=1 / 2$, the gel cannot absorb or release any solvent and the final and initial state are identical. In that case, the inverse Laplace transform can be performed analytically and the deflection is $\hat{\zeta}(s, t)=\hat{\zeta}^{i}(s) H(t)$. This solution is the purely elastic response originally derived by Jerison and later by Style and Dufresnes. The displacement of the interface at large distances (small wavenumber $s$ ) behaves as $\sim \hat{f}_{z}(s) /(2 G s)$ and the deflection is thus damped by the bulk elasticity. At small distances (large $s$ ), the deflection behaves as $\sim \hat{f}_{z}(s) /\left(s^{2} \gamma_{s}\right)$ and is thus dominated by surface tension. The crossover between the elastic and the capillary regime occurs at the scale of the elastocapillary length $\ell_{s}$.

\section{F. General solution for arbitrary force distribution}

The fundamental solution (30) derived above can be exploited to generate the impulsional response of the system to a forcing of the form $\vec{f}=f_{z}(r) \delta(t) \vec{e}_{z}$ (by taking the derivative) or convolved to generate the response to more general time-dependent forcing $\vec{f}=f_{z}(r) F(t) \vec{e}_{z}$. Indeed, 
using Duhamel principle of superposition, the solution $\xi(r, t)$ to this arbitrary time dependent forcing can be written as in term of the step response $\zeta(r, t)$ given in equation (30):

$$
\xi(r, t)=F\left(0^{+}\right) \zeta(r, t)+\int_{0}^{t} \zeta(r, \tau) \frac{\partial F(t-\tau)}{\partial t} \mathrm{~d} \tau
$$

This general solution will be investigated at the end of the next section but we first turn to the analysis of the solution (30) in the case of a hemispherical droplet on a poroelastic substrate.

\section{RESULTS FOR HEMISPHERICAL DROPLETS}

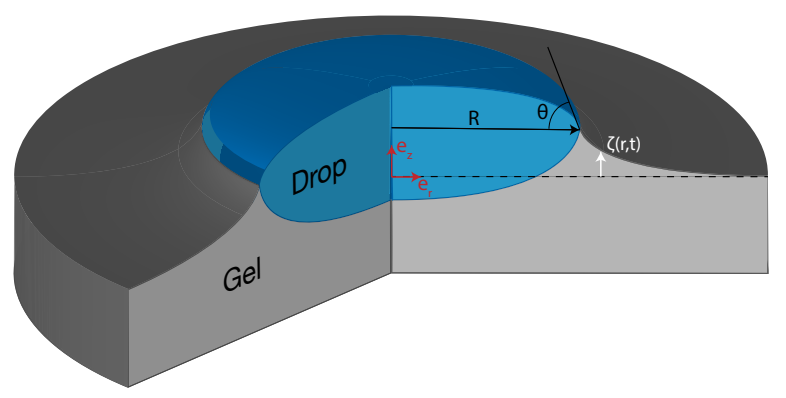

Figure 1. Schematic representation of the problem and notations. A liquid hemispherical droplet with radius $R$ and contact angle $\theta$ is deposited at time $t=0$ at the surface of a poroelastic substrate. The black dashed line indicates the position of the free surface before the deposition of the liquid drop. Using cylindrico-polar coordinates $(r, z)$ in the frame $\left(\vec{e}_{r}, \vec{e}_{z}\right)$, the deflection of the free surface is given by the function $\zeta(r, t)$.

We now consider the specific case where the surface force distribution is due to a hemispherical droplet with radius $R$ and contact angle $\theta$. In this situation, the surface force distribution is $f_{z}(r)=$ $\gamma \sin \theta \delta(r-R)-2 \frac{\gamma \sin \theta}{R} H(R-r)$. The first term is due to the liquid/air surface tension that pull on the substrate at the contact line while the second term is due to the Laplace pressure inside the spherical droplet that push the substrate. The zeroth-order Hankel transform of $f_{z}(r)$ is:

$$
\hat{f}_{z}(s)=\gamma \sin \theta\left(R J_{0}(s R)-\frac{2 J_{1}(s R)}{s}\right)
$$

With this choice of surface force distribution, the interface profile $\zeta(r, t)$ is given by: 


$$
\zeta(r, t)=\frac{\gamma \sin \theta}{\gamma_{s}} \ell_{s} \int_{\xi-i \infty}^{\xi+i \infty} \mathrm{d} \omega \int_{0}^{\infty} \mathrm{d} s \frac{e^{\omega t} J_{0}(s r)\left(R J_{0}(s R)-\frac{2 J_{1}(s R)}{s}\right)}{\omega\left(1+s \ell_{s}\right)+s^{2} D^{\star} \frac{1-2 v}{1-v}\left(\frac{s \sqrt{D^{\star}}}{\sqrt{s^{2} D^{\star}+\omega}}-1\right)}
$$

We now turn to the detailed analysis of equation (35) for specific cases of broad physical interest.

\section{A. Large drops}

The first limiting case of interest is the case of large drops, i.e $R \gg \ell_{s}$. We plot in Fig.2 the time evolution of the surface deformation for a large drop $\left(R / \ell_{s}=100\right)$, as well as the associated concentration and chemical potential fields. As seen in Fig.2-B the lower part of the drop sinks over time inside the soft substrate. The ridge height $h(t)=\zeta(R, t)$ on the other hand, increases in a non-trivial fashion after the initial deposition and its evolution is plotted in Fig.2-C. Before the deposition, the interface is flat $h\left(0^{-}\right)=0$. Right after the deposition, the height suddenly jumps to a height $h\left(0^{+}\right)$. Asymptotically, for large drops, the height $h\left(0^{+}\right)$of the ridge is given by:

$$
h\left(0^{+}\right) \approx \frac{\gamma \sin \theta}{\gamma_{s}} \ell_{s} \frac{\Gamma_{e}-4+\log \frac{8 R}{\ell_{s}}}{\pi}
$$

where $\Gamma_{e}$ is the Euler-Mascheroni constant. Following this initial jump, the height of the ridge increases as the solvent migrates toward the ridge where the gel is under tension. In the same time, the ridge moves radially toward the interior of the drop up to a distance of $\ell_{s}(\gamma \sin \theta(1-2 v)) /\left(4 \gamma_{s}\right)$ in the final state. Because this time evolution is due to the diffusive migration of the solvent on a distance of order $R$, the stationary state is reached on a timescale of $\sim R^{2} / D^{\star}$, as can be seen in the inset of Fig.2-C. Quite surprisingly for such a damped system, the ridge height first increases above its final stationary value $h(\infty)$ before relaxing toward $h(\infty)$ which is given asymptotically by:

$$
h(\infty) \approx \frac{\gamma \sin \theta}{\gamma_{s}} \ell_{s} \frac{2(1-v)\left(\Gamma_{e}-4+\log \frac{4 R}{\ell_{s}(1-v)}\right)}{\pi}
$$

This non-trivial overshooting behavior can be understood by analyzing the two forces that are applied to the surface of the poro-elastic substrate. While both forces imply a migration of solvent over a lengthscale $R$, the Laplace pressure in the drop acts as a distributed pressure on the surface that pushes fluid only in the outward radial direction. On the other hand, the traction due to the 

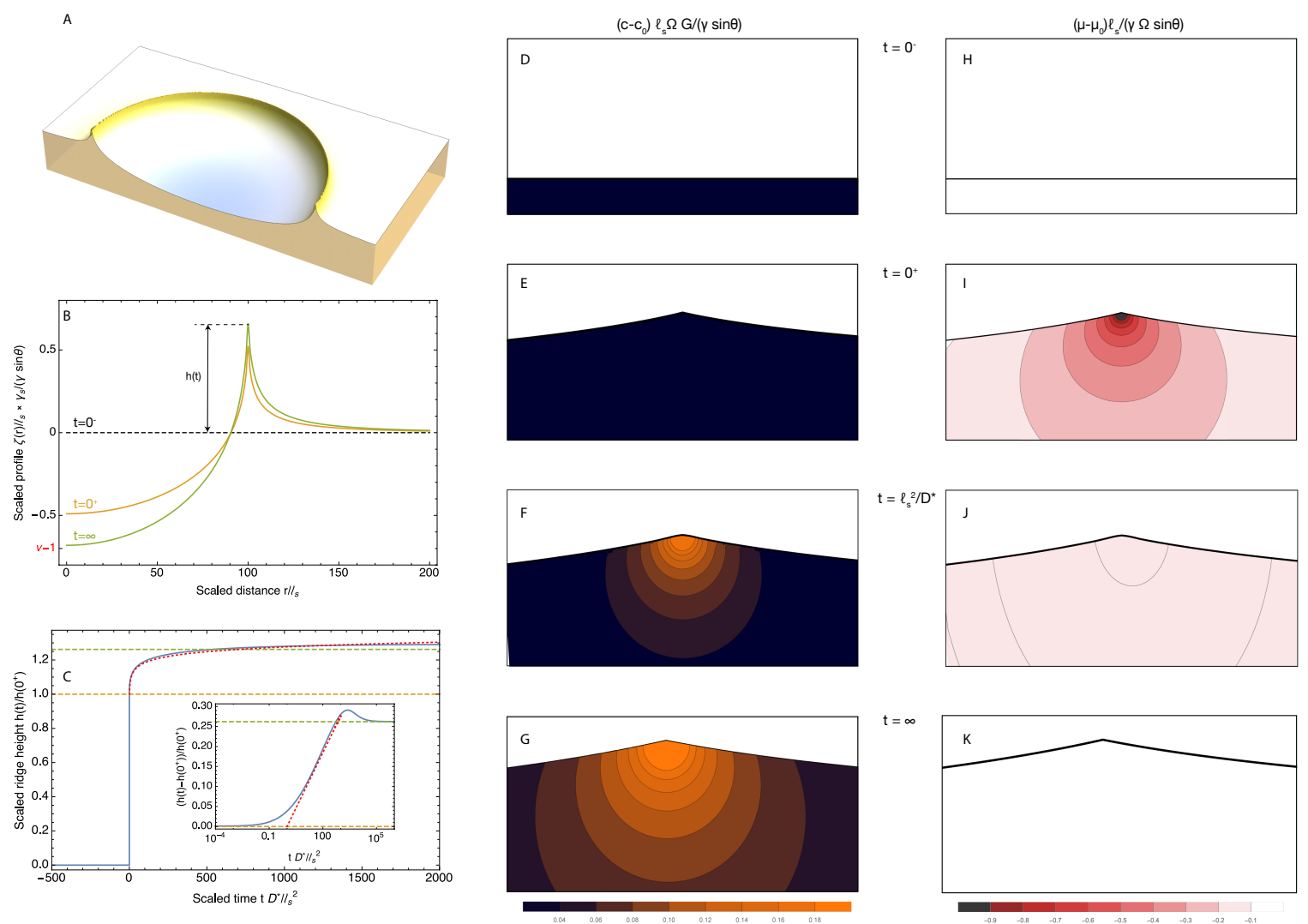

Figure 2. Time dependent deformation of the poroelastic substrate following the deposition of a large drop $\left(R / \ell_{s}=100\right)$ and a poroelastic Poisson ratio of 3/10. A: 3D view of a half space deformed by the drop showing the ridge at the triple line. The drop is not drawn for clarity. B: Dimensionless profile of the interface $\zeta(r, t) / \ell_{s}$ at $t=0^{+}$(orange curve) and $t=\infty$ (green curve). The initial position of the interface before deposition at $t=0^{-}$is indicated by a black dotted curve. C: Time evolution the height of the ridge (solid blue curve), scaled by its instantaneous value $h\left(0^{+}\right)$. The instantaneous response is indicated by an orange dashed line while the final equilibrium value is indicated by a green dashed line. The asymptotic law $h(t)-h(0+) \propto \ell_{s} \log \left(t D^{\star} / \ell_{s}^{2}\right)$ is shown by a red dotted curve. Shown in inset is the scaled poroelastic response $(h(t)-h(0+)) / h(0+)$ after deposition with a log scale for the time coordinate, clearly showing the overshoot behavior discussed in the text. D-G: Evolution of the dimensionless solvent concentration field $\left(c-c_{0}\right) \Omega$ before the deposition of the drop at $t=0^{-}(\mathbf{D})$, right after deposition at $t=0^{+}(\mathbf{E})$, at $t=\ell_{s}^{2} / D^{\star}(\mathbf{F})$ and finally at $t=\infty(\mathbf{G})$. H-K: Evolution of the dimensionless chemical potential field $\left(\mu-\mu_{0}\right) G \Omega$ before the deposition of the drop at $t=0^{-}$(D), right after deposition at $t=0^{+}(\mathbf{E})$, at $t=\ell_{s}^{2} / D^{\star}(\mathbf{F})$ and finally at $t=\infty(\mathbf{G})$. In D-K, the concentration and chemical potential fields are plotted in regions centered at $R / \ell_{s}=100$ and have width and height of $2 \ell_{s}$ and $\ell_{s}$, respectively. 
air/liquid interface is a force localized at the triple line and draws fluid from both the inside and the outside of the drop. As a consequence the increase in height due to this traction relaxes twice as fast as the decrease in height due to the Laplace pressure. The combination of these two forces with slightly different timescales therefore produces the overshoot behavior seen in the inset of Fig.2-C.

Because the inverse Hankel-Laplace cannot be evaluated analytically, it is not possible to provide a simple expression (in the time domain) for the time evolution of the ridge height $h(t)$. However, some crude approximations can be performed in order to gain further insight on the behavior of $h(t)$. In the limit of large drop, we focus on the evolution of $h(t)$ between the two intermediate timescales $\ell_{s}^{2} / D^{\star} \ll t \ll R^{2} / D^{\star}$ and we will make the crude approximation that the evolution of $h(t)$ in this regime is mostly due the evolution of the corresponding lenghtscales $1 / R \ll s \ll 1 / \ell_{s}$ and we will check later that this approximation is self-consistent. In this limit, the Laplace transform of the increase of the ridge height $h(t)-h(0+)$ is then approximately $\ell_{s} \int_{1 / R}^{1 / \ell_{s}} s /\left\{\omega\left(s^{2}-\left(\omega / D^{\star}\right)(1-v) /(1-2 v)\right)\right\} \mathrm{d} s$. This simpler expression can then be integrated along $s$ and the resulting expression can finally be inverted in the time domain analytically to yield the scaling

$$
h(t)-h(0+) \propto \ell_{s} \log \left(t D^{\star} / \ell_{s}^{2}\right)
$$

As seen in Fig.2-C, this expression fits rather well the numerical result between the two intermediate timescales $\ell_{s}^{2} / D^{\star} \ll t \ll R^{2} / D^{\star}$, as expected from our assumptions. Beside providing a reasonable approximation to the evolution of the ridge height, it also shows that the relevant timescale for the evolution of the ridge created by large drops is $\ell_{s}^{2} / D^{\star}$. Beneath the drop, the depth of the valley is, at leading order, independent of the drop size and increases over time, from $\zeta\left(r=0, t=0^{+}\right) \approx-\frac{\ell_{s}}{2} \frac{\gamma \sin \theta}{\gamma_{s}}$ until it reaches $\zeta(r=0, t=\infty) \approx-(1-v) \ell_{s} \frac{\gamma \sin \theta}{\gamma_{s}}$. As seen in Fig.2$\mathrm{B}$, the formula above are a good approximation for the case $R / \ell_{s}=100$. Beneath the drop, the chemical potential increases right after the deposition. We find that, for large drops, the chemical potential beneath the drop at $t=0^{+}$is given by:

$$
\mu\left(r=0, z=0, t=0^{+}\right) \approx \mu_{0}+2 \frac{\gamma \sin \theta \Omega}{R}
$$

At the contact line however, the chemical potential diverges as $\log |r-R|$. In the final state, the chemical potential relaxes everywhere to $\mu_{0}$. Similarly, the concentration of solvent, initially 
equal to $c_{0}$ reaches the following value beneath the drop in the steady state.

$$
c(r=0, z=0, t=\infty) \approx c_{0}-2(1-2 v) \frac{\gamma \sin \theta}{G \Omega R}
$$

As can be seen from equations (39) and (40), although the depth of the valley increases over time, the change in concentration beneath the drop is very small. On the other hand, and while the change in amplitude of the ridge is of the same order than that of the depth of the valley, the solvent concentration increases sharply (it also diverges as $\log |r-R|$ ) beneath the ridge. We therefore only plot here the concentration (Fig.2 D-G) and chemical potential (Fig.2 H-K) fields in the vicinity of the contact line. As seen in those panels, the solvent concentration field $\left(c-c_{0}\right)$ is zero at $t=0^{+}$but then increases sharply near the contact line where we also notice the radial (inward) displacement of the triple line over time.

\section{B. Small drops}

We now turn to the analysis of equation (35) for a second case where analytical approximations are possible, namely, small drops $\left(R \ll \ell_{S}\right)$. We plot in Fig.3-B the evolution of the surface deformation at various time for a small drops $\left(R / \ell_{s}=1 / 100\right)$. At the time of deposition, small drops adopt a lenticular shape and the substrate is flat outside of the drop. This is due to the fact that below the elasto-capillary length, all perturbations are damped by capillarity. Consequently, both the Laplace pressure and the surface tension at the contact line are balanced solely by the surface tension of the gel and not by its elasticity. As only surface tensions play a role in the force balance in this case, the situation is analogous to that of a liquid lens on a liquid bath, hence the lenticular shape. Indeed, beneath the drop, the depth of the valley is, at leading order, independent of time and given by:

$$
\zeta\left(r=0, t=0^{+}\right) \approx \zeta(r=0, t=\infty) \approx-\frac{\ell_{s}}{4} \frac{\gamma_{\sin }(\theta)}{\gamma_{s}}\left(\frac{R}{\ell_{s}}\right)
$$

As the consequence of the lenticular shape, we therefore expect the ridge height to be asymptotically zero at leading order. Right after the deposition, the height suddenly jumps to a small height $h\left(0^{+}\right)$which, for small drops is given by the first non-zero term of an expansion in $R / \ell_{s}$ :

$$
h\left(0^{+}\right) \approx \frac{\gamma \sin (\theta)}{\gamma_{s}} \ell_{s} \frac{4}{9 \pi}\left(\frac{R}{\ell_{s}}\right)^{2}
$$



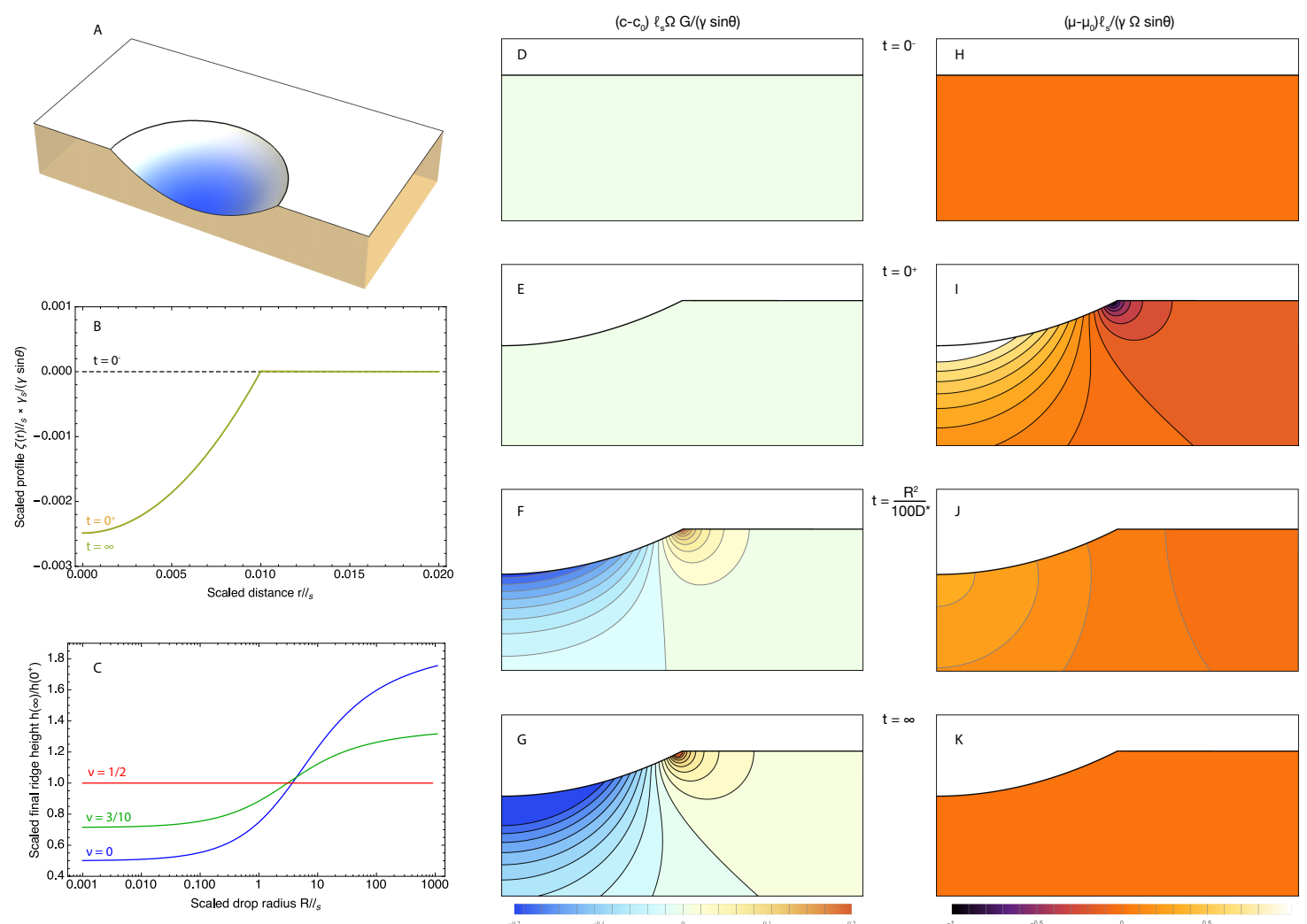

Figure 3. Time dependent deformation of the poroelastic substrate following the deposition of a small drop $\left(R / \ell_{s}=1 / 100\right)$ and a poroelastic Poisson ratio of 3/10. A: 3D view of a half space deformed by the drop showing the ridge at the triple line. The drop is not drawn for clarity. B: Dimensionless profile of the interface $\zeta(r, t) / \ell_{s}$ at $t=0^{+}$(orange curve) and $t=\infty$ (green curve). The initial position of the interface before deposition at $t=0^{-}$is indicated by a black dotted curve. To first order in $R / \ell_{s}$ the shape of the deformed gel is unchanged. C: Scaled value of the final ridge height $h(\infty) / h\left(0^{+}\right)$as a function of the scale radius of the drop $R / \ell_{s}$ for three different values of the poroelastic Poisson ratio ( $v=1 / 2$ : red curve, $v=3 / 10$ : green curve, $v=0$ : blue curve). D-G: Evolution of the dimensionless solvent concentration field $\left(c-c_{0}\right) \ell_{s} \Omega G /(\gamma \sin \theta)$ before the deposition of the drop at $t=0^{-}$(D), right after deposition at $t=0^{+}(\mathbf{E})$, at $t=R^{2} / 10 D^{\star}$ (F) and finally at $t=\infty(\mathbf{G})$. H-K: Evolution of the dimensionless chemical potential field $\left(\mu-\mu_{0}\right) \ell_{s} /(\gamma \Omega \sin \theta)$ before the deposition of the drop at $t=0^{-}$ (D), right after deposition at $t=0^{+}(\mathbf{E})$, at $t=R^{2} / 10 D^{\star}(\mathbf{F})$ and finally at $t=\infty(\mathbf{G})$. In D-K, the concentration and chemical potential fields are plotted in regions centered at $R / \ell_{s}=1 / 100$ and have width and height of $2 R$ and $R$, respectively. 
After relaxation, the ridge height reaches $h(\infty)$ which is given asymptotically by:

$$
h(\infty) \approx \frac{\gamma \sin (\theta)}{\gamma_{s}} \ell_{s} \frac{2}{9 \pi(1-v)}\left(\frac{R}{\ell_{s}}\right)^{2}
$$

We note that the ridge height is now quadratic in $R / \ell_{s}$. The consequence of this is that, at first order in $R / \ell_{s}$, and in stark contrast with large drop, the profile is flat outside the drop. To the same order the profile is thus independent of time for small drop, as can be seen in Fig.3-B. This effect could in fact be expected since the shape of the substrate deformation is controlled solely by capillarity for small drops. As it is therefore independent of the mechanical property of the substrate (again, to first order in $R / \ell_{s}$ ), the profile is both independent of the shear modulus and the poroelastic Poisson ratio, i.e of the ability of the substrate to reorganize the solvent. We note that, in opposition with large drops, the ratio $h(\infty) / h\left(0^{+}\right)$is smaller than 1 and thus small drops gradually sink inside the gel although this is a second order effect in $R / \ell_{s}$. This can also be seen on Fig.3-C that shows the ratio $h(\infty) / h\left(0^{+}\right)$as a function of the drop size $R \ll \ell_{s}$ for different value of the poroelastic ratio $v$. Note that since $R \ll \ell_{s}$, the relevant timescale for the evolution of the ridge profile is not $\ell_{s}^{2} / D^{\star}$ anymore, as was the case for large drops, but $R^{2} / D^{\star}$. Now if the shape of the substrate is, at leading order, independent of time, how does the solvent evolve? Beneath the drop, the chemical potential increases right after the deposition while it drops under the ridge. Asymptotically, we find that the chemical potential beneath the drop is given by:

$$
\mu\left(r=0, z=0, t=0^{+}\right) \approx \mu_{0}+\frac{\gamma \sin (\theta) \Omega}{\ell_{s}}
$$

As the chemical potential converge to $\mu_{0}$ away from the drop, there is indeed a gradient of chemical potential that drive fluid motion. In the final state, the concentration of solvent beneath the drop is given by:

$$
c(r=0, z=0, t=\infty) \approx c_{0}-\frac{1-2 v}{2(1-v)} \frac{\gamma \sin (\theta)}{G \ell_{S} \Omega}
$$

The corresponding concentration and chemical potential are plotted in Fig.3 D-G and Fig.3 $\mathrm{H}-\mathrm{K}$, respectively. 


\section{Drop removal}

We now investigate the effect of removing the drop at the free surface after a residence time $\tau_{\text {res. }}$. We therefore now have a forcing of the form $\vec{f}=\left\{f_{z}(r) H(t)-f_{z}(r) H\left(t-\tau_{\text {res }}\right)\right\} \vec{e}_{z}$. Using the convolution integral given in equation (35), the solution $\zeta_{\mathrm{res}}(r, t)$ describing the profile of the interface for the deposition/removal problem is simply given by:

$$
\zeta_{\text {res }}(r, t)=\zeta(r, t)-H\left(t-\tau_{\text {res }}\right) \zeta\left(r, t-\tau_{\text {res }}\right)
$$

where $\zeta(r, t)$ is given by (35). As the ridge is pronounced only for $R \gg \ell_{S}$, we focus here on large drops only. We plot in Fig.4-A the profile of the poroelastic substrate for a residence time $\tau_{\text {res }}=10 \ell_{s}^{2} / D^{\star}$ at various time following the removal of the drop. These curves indicate that the drop leaves a footprint on the gel that slowly relaxes to a flat interface. More quantitatively, and as seen on Fig. 4, the height of the ridge drops by an amount $h\left(0^{+}\right)$immediately following the removal of the drop, at time $\tau_{\text {res }}^{+}$, i.e $h\left(\tau_{\text {res }}^{+}\right)=h\left(\tau_{\text {res }}^{-}\right)-h\left(0^{+}\right)$. Following this instantaneous elastic response, the height then relaxes toward zero as the solvent diffuses back to its original concentration $c=c_{0}$ : this is the poroelastic response. We plot in the inset of Fig.4-B the relaxation of the ridge for several values of the residence time $\tau_{\text {res. }}$. As seen in Fig.4-C, the relaxation depends on the history of the gel and is faster for smaller residence time $\tau_{\text {res }}$. For residence time $\tau_{\text {res }}$ smaller than the timescale $\ell_{s}^{2} / D^{\star}$ the ridge height decreases as $\sim 1 / t$ at intermediate timescales $\ell_{s}^{2} / D^{\star} \ll t \ll R^{2} / D^{\star}$. However, for residence time $\tau_{\text {res }}$ larger than the timescale $\ell_{s}^{2} / D^{\star}$, the ridge height decrease more slowly, as $\sim-\log (t)$. In order to estimate the lifetime of the drop footprint, we define a time $\tau_{\text {life }}$ which correspond to the time it takes for the deformation to reach a critical thickness $h_{c}$, i.e we defined $\tau_{\text {life }}$ as the solution of the equation:

$$
\zeta_{\text {res }}\left(r, \tau_{\text {life }}\right)=h_{c}
$$

In the present study, the value of this critical thickness is of course arbitrary but it can be quantified for specific applications. In the context of wetting for example, surface defects as small as $10 \mathrm{~nm}$ can pin the contact line and affect the static equilibrium angle. As a consequence, if the footprint of a drop is thicker than this critical thickness, it will have consequences at the macroscopic scale on the wetting properties of the gel for instance. As seen in Fig.4-D, the lifetime of the drop footprint strongly depends on this critical thickness and shows a non-trivial dependence on the residence time $\tau_{\text {res }}$ of the drop. We first note that the residence time must be larger than a 

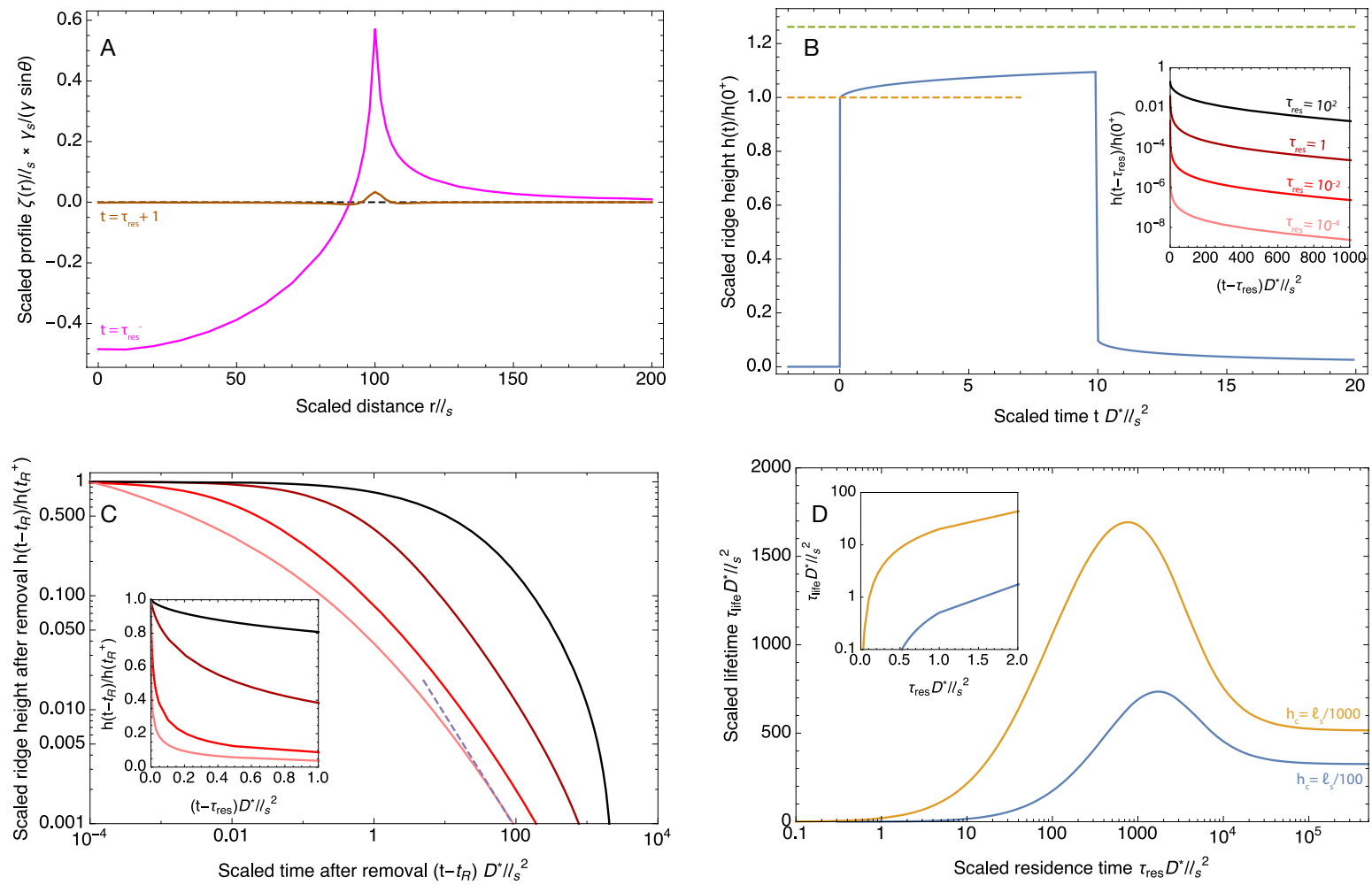

Figure 4. Time dependent deformation of the poroelastic substrate following the removal of a large drop $\left(R / \ell_{s}=100\right)$ and a poroelastic Poisson ratio of $3 / 10$. A: Profile of the interface at $t=\tau_{\text {res }}^{-}$ (magenta curve) and $t=\tau_{\text {res }}+\ell_{s}^{2} / D^{\star}$ (brown curve). The initial position of the interface before deposition at $t=0^{-}$is indicated by a black dotted curve. B: Time evolution the height of the ridge (solid blue curve), scaled by its instantaneous response at the time of deposition $h\left(0^{+}\right)$. The instantaneous response is indicated by an orange dashed line while the final equilibrium value is indicated by a green dashed line. The drop is removed at $t=\tau_{\text {res }}=10 \ell_{s}^{2} / D^{\star}$. The height of the ridge first elastically decrease instantaneously by an amount of $h\left(0^{+}\right)$before relaxing poroelastically. Several scaled $h\left(t-\tau_{\text {res }}\right) / h\left(0^{+}\right)$profiles of the poroelastic relaxation are shown in inset for different values of the residence time $t=\tau_{\text {res. }}$. The residence times are given in units of $D^{\star} / \ell_{s}^{2}$. C: $\log -\log$ plots of the scaled poroelastic profiles $h\left(t-\tau_{\text {res }}\right) / h\left(\tau_{\text {res }}^{+}\right)$as a function of the scaled time after removal $\left(t-\tau_{\text {res }}\right) D^{\star} / \ell_{s}^{2}$ for different values of the residence time. A close view in linear scale is given in inset. D: Scaled lifetime of the footprint of the drop $\tau_{\text {life }} D^{\star} / \ell_{s}^{2}$ as a function of the scaled residence time of the drop $\tau_{\text {res }} D^{\star} / \ell_{s}^{2}$ for two different values of the critical thickness $h_{c}$ below which the footprint disappears.

critical value for the height of the footprint to be larger than $h_{c}$. This effect can be seen in the inset 
of Fig.4-D. Above this critical residence time, the footprint lifetime $\tau_{\text {life }}$ first increases with the residence time $\tau_{\text {res }}$ until the residence time becomes comparable with the equilibrium time $R^{2} / D^{\star}$. After this value, the lifetime decreases. This decrease is simply the consequence of the overshoot effect described previously for the growth of the ridge. When the residence time is much larger than $R^{2} / D^{\star}$, then the gel has reached its equilibrium before the drop is removed. In that case, the lifetime of the footprint does not depend on the residence time of the drop and therefore $\tau_{\text {life }}$ saturates to a finite value.

\section{DISCUSSION}

In typical experiments of elastowetting, $\ell_{s}$ is typically of order of a few microns but can be as large as a millimeter thanks to recent advances on polymer technologies. The effective diffusion coefficient $D^{\star}$ is typically in the range $10^{-11}-10^{-10} \mathrm{~m}^{2} / \mathrm{s}$ depending on the swelling ratio and the length of the free chains ${ }^{49,50}$ while values in the range $0.2-0.4$ are reported for the poroelastic Poisson ratio. For such orders of magnitude of the physical parameters, the deformation created by a $1 \mathrm{~mm}$ droplet will take as long as $\sim 10^{5}$ s to reach its equlibrium state. More quantitatively, for an elastocapillary length of $10 \mu \mathrm{m}$, a $1 \mathrm{~mm}$ drop with a residence time of 20s will leave a footprint thicker than $100 \mathrm{~nm}$ for also roughly 20 s and it will leave a footprint thicker than 10nm for more than 7 minutes. Similarly, a drop resting on a poroelastic substrate for 10 minutes will leave a circular footprint thicker than 10nm for more than 2 hours. Preliminary experiments performed with water droplets on thick PDMS layers are in qualitative agreement, as seen in Fig.5, with our predictions. Because nanometer-scales defects can pin a contact line, we expect that the residence time of the drop on the deformable substrate will have a dramatic impact on the measurement of fundamental material properties of considerable practical interest, such as its surface tension or contact angle hysteresis. Those footprints will also affect drop spreading. In addition, because nanoscale surface features are strong enough to affect the polarity and the migration of

living cells, ${ }^{51-54}$ we expect that the new theoretical developments presented in this paper will be important to finely model the locomotion of cells in living tissues and on soft materials.

In our study, we have also seen that another consequence of merging the linear poroelastic theory with the elastowetting problem has introduced a new divergence: the solvent concentration diverges as $\sim \log |r-R|$ near the contact line. While several approaches might be able to regularize this divergence, for example by taking into account the finite thickness of the gel, the material and 

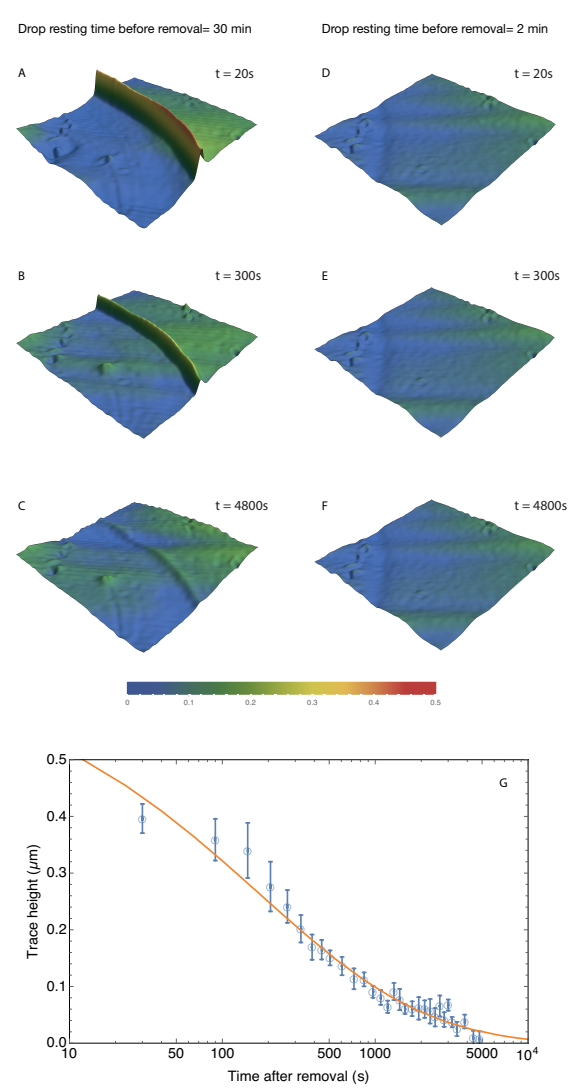

Figure 5. Relaxation of the ridge under the contact line following the removal of the drop for a resting time of 30 minutes (A-C) and 2 minutes (D-F). In both cases $5 \mu \mathrm{L}$ water droplets were placed on a freshly prepared thick PDMS substrate (thickness $1358 \mu \mathrm{m}$, shear modulus $1.2 \mathrm{kPa}$ ) and a region of $\sim 750 \times 750 \mu \mathbf{m}^{2}$ was scanned at regular intervals with a 3D profiler (Microsurf 3D, Fogal Nanotech, France) with a lateral resolution of $1.89 \mu \mathrm{m}$ and a vertical resolution of $50 \mathrm{~nm}$. The first scan was acquired roughly 20 s following the removal of the drops. The color-coded heights are in microns. As seen in D-F, no trace could be detected for a residence time of 2 minutes. For a residence time of 30 minutes on the other hand (A-C), the footprint of the drop is initially around $0.5 \mu \mathrm{m}$ and and is still around $100 \mathrm{~nm}$ after $4800 \mathrm{~s}$. G: Time evolution of the height of the trace following drop removal (same parameters as in A-C). The blue circles are the experimental data and the solid orange curve is the theoretical prediction given by equation (46). For the theoretical curve, the droplet radius and substrate stiffness were taken from the experiment while the surface tension was taken as $\gamma_{s}=40 \mathrm{mN} / \mathrm{m}$ and the poroelastic Poisson Ratio was taken as $v=0.3$. The effective diffusion coefficient $D^{\star}$ was found by fitting the data with the model. The best fit for $D^{\star}$ was found to be $\sim 2 \cdot 10^{-11} \mathbf{m}^{2} / \mathbf{s}$, in good agreement with other values found in the literature. 
geometrical nonlinearities or through the introduction of a finite width for the contact line, the existence of this divergence suggests that extreme phenomena such as phase separation or fracture could occur at the contact line. Indeed, the coexistence of multiple phases at the contact line has been recently reported in indentation experiments. Further work, for example based on a nonlinear poroelastic theory, will be needed in order to shed light on the behavior of gels near contact lines.

In a different line of thought, the wetting of saturated gels by drops of their own solvent opens interesting questions. Because the presence of a drop change the chemical potential away from the drop, several drops may interact with each other by mass exchange throughout the gel. For thick gels (when the thickness of the gel is much larger than $\ell_{S}$ and $R$ ), the chemical potential increases above its reference value away from the drop and will tend to suck fluid inside the gel. Because large drops will create a stronger change in chemical potential than small drops we thus expect large drops to grow at the expanse of smaller droplets. For thinner gels however, the effect of finite depth is likely to form a dimple within which the chemical potential drops below its reference value, and thus promote the growth of smaller droplets nearby. Although speculative, this possibility might open the road to new original methods to control droplet nucleation and dew collection on soft materials.

[1] Sokuler, M. et al. The softer the better: Fast condensation on soft surfaces. Langmuir 26, 1544?1547 (2010).

[2] Unger, M. A., Chou, H.-P., Thorsen, T., Scherer, A. and Quake, S. R. Monolithic Microfabricated Valves and Pumps by Multilayer Soft Lithography. Science (80-. ). 288, 113 ?116 (2000).

[3] Boland,T.,Xu,T.,Damon,B. and Cui,X. Application of inkjet printing to tissue engineering.Biotechnol. J. 1, 910 ?917 (2006).

[4] Chung, S. et al. Inkjet-printed stretchable silver electrode on wave structured elastomeric substrate. Appl. Phys. Lett. 98, 2011 ?2014 (2011).

[5] Lester, G. R. Contact angles of liquids at deformable solid surfaces. J. Colloid Sci. 16, 315 ?326 (1961).

[6] Lester, G. R. Contact angles of liquids on organic solids. Nature 209, 1126 ?1227 (1966).

[7] Shanahan, M. E. R. Contact Angle Equilibrium on Thin Elastic Solids. J. Adhes. 18, 247?267 (1985).

[8] Shanahan, M. E. R. and de Gennes, P. G. L?arête produite par un coin liquide près de la ligne triple de contact solide/liquide/fluide. C. R. Acad. Sci. Paris 302, $517 ? 521$ (1986). 
[9] Shanahan, M. E. R. The influence of solid micro-deformation on contact angle equilibrium. J. Phys. D 20, $945 ? 950$ (1987).

[10] Long, D., Ajdari, A. and Leibler, L. Static and dynamic wetting properties of thin rubber films. Langmuir 12, $5221 ? 5230$ (1996).

[11] Yu, Y.-S., Yang, Z. and Zhao, Y.-P. Role of vertical component of surface tension of the droplet on the elastic deformation of PDMS membrane. J. Adhes. Sci. Technol. 22, $687 ? 698$ (2008).

[12] Yu,Y.S.and Zhao,Y.P. Elastic deformation of soft membrane with finite thickness induced by a sessile liquid droplet. J. Colloid Interface Sci. 339, 489 ?494 (2009).

[13] Pericet-Camara, R. et al. Solid-supported thin elastomer films deformed by microdrops. Soft Matter 5, $3611 ? 3617$ (2009).

[14] Roman, B.and Bico, J. Elasto-capillarity: deforming an elastic structure with a liquid droplet. J. Phys. Condens. Matter 22, 493101 (2010).

[15] Limat, L. Straight contact lines on a soft, incompressible solid. EPJ E 35, 134?147 (2012).

[16] Style, R. W. and Dufresne, E. R. Static wetting on deformable substrates, from liquids to soft solids. Soft Matter 8, 7177 (2012).

[17] Style, R. W. et al. Universal deformation of soft substrates near a contact line and the direct measurement of solid surface stresses. Phys. Rev. Lett. 066103 (2013).

[18] Style, R. W., Hyland, C., Boltyanskiy, R., Wettlaufer, J. S. and Dufresne, E. R. Surface tension and contact with soft elastic solids. Nat. Commun. 4, 2728 (2013).

[19] Karpitschka, S. et al. Droplets move over viscoelastic substrates by surfing a ridge. Nat. Commun. 6, $7891(2015)$

[20] Style,R.W. et al.Patterning droplets with durotaxis. Proc. Natl. Acad. Sci. 110,12541?12544(2013).

[21] Park, S. J. et al. Visualization of asymmetric wetting ridges on soft solids with X-ray microscopy. Nat. Commun. 5, 4369 (2014).

[22] K. E. Jensen, R. Sarfati, R. W. Style, R. Boltyanskiy, A. Chakrabarti, M. K. Chaudhury, E. R. Dufresne Wetting and phase separation in soft adhesion. Proc. Natl. Acad. Sci. U.S.A. 112, 14490-14494 (2015).

[23] Zhao, M. et al. Thickness effects in the wetting of soft solids. Submitt. (2017).

[24] Dervaux,J. and Limat,L. Contact lines on soft solids with uniform surface tension: analytical solutions and double transition for increasing deformability. Proc. R. Soc. A 471, 20140813 (2015).

[25] De Pascalis R, Dervaux J, Ionescu I and Limat L. Nonlinear elastowetting: multiscale modelling of the triple line (submitted) 
[26] Hong W, Zhao X, Zhou J, Suo Z. 2008. J. Mech. Phys. Solids 56:1779-93

[27] Hong W, Liu Z, Suo Z. 2009. Int. J. Solids Struct. 46:3282-89.

[28] Hui CY, Muralidharan V. 2005. J. Chem. Phys. 123:154905

[29] Y. Hu and Z. Suo. Viscoelasticity and poroelasticity in elastomeric gels. Acta Mechanica Solida Sinica, 25:441-458, 2012.

[30] J.R. Rice and M.P. Cleary. Some basic strss diffusion solutions for fluid -saturated elastic porous media with compressible constituents. Reviews of Geophysics and Space Physics, 14:227-241, 1976.

[31] H.F. Wang. Theory of Linear Poroelasticity with Ap- plications to Geomechanics and Hydrogeology. Princeton University Press, 2000.

[32] Biot MA. 1941. J. Appl. Phys. 12:155-64

[33] Roberts J. J., Earnshaw A., Ferguson V. L., Bryant S. J. 2011 Comparative study of the viscoelastic mechanical behavior of agarose and poly(ethylene glycol) hydrogels. J. Biomed. Mater. Res. B 99B, 158-169.

[34] Liu Y., Chan-Park M. B. 2009 Hydrogel based on interpenetrating polymer networks of dextran and gelatin for vascular tissue engineering. Biomaterials 30, 196-207.

[35] Jeong-Yun Sun, Xuanhe Zhao, Widusha R.K. Illeperuma, Kyu Hwan Oh, David J. Mooney, Joost J. Vlassak, Zhigang Suo. Highly stretchable and tough hydrogels. Nature. 2012 Sep 6; 489(7414): 133136.

[36] Ferry, J.D.: Viscoelastic Properties of Polymers. John Wiley \& Sons, Inc., New York (1980)

[37] Zhao, X., Huebsch, N., Mooney, D.J., et al.: Stress-relaxation behavior in gels with ionic and covalent crosslinks. Journal of Applied Physics 107, 063509 (2010)

[38] Chen, D.T., Wen, Q., Janmey, P.A., et al.: Rheology of soft ma- terials. Annual Review of Condensed Matter Physics 1, 301- 322 (2010)

[39] Hu, Y., Chen, X., Whitesides, G.M., et al.: Indentation of poly- dimethylsiloxane submerged in organic solvents. J. Mater. Res. 26, 785-795 (2011)

[40] Kalcioglu, Z.I., Mahmoodian, R., Hu, Y., et al.: From macro-to microscale poroelastic characterization of polymeric hydrogels via indentation. Soft Matter 8, 3393-3398 (2012)

[41] Strange, D.G., Fletcher, T.L., Tonsomboon, K., et al.: Sepa- rating poroviscoelastic deformation mechanisms in hydrogels. Appl. Phys. Lett. 102, 031913 (2013)

[42] Hyland, L.L., Taraban, M.B., Feng, Y., et al.: Viscoelastic properties and nanoscale structures of composite oligopeptide- polysaccharide hydrogels. Biopolymers 97, 177-188 (2012) 
[43] Olberding, J.E., Francis Suh, J.: A dual optimization method for the material parameter identification of a biphasic poro- viscoelastic hydrogel: Potential application to hypercompliant soft tissues. J. Biomech. 39, 2468-2475 (2006)

[44] Wang, X., Hong, W.: A visco-poroelastic theory for polymeric gels. Proceedings of the Royal Society A: Mathematical, Phys- ical and Engineering Science 468, 3824-3841 (2012)

[45] Biot, M.: Theory of deformation of a porous viscoelastic anisotropic solid. Journal of Applied Physics 27, 459-467 (1956)

[46] Weitsman, Y.: Stress assisted diffusion in elastic and viscoelas- tic materials. Journal of the Mechanics and Physics of Solids 35, 73-94 (1987)

[47] Weitsman, Y.: A continuum diffusion model for viscoelastic materials. Journal of Physical Chemistry 94, 961-968 (1990)

[48] S. Karpitschka, S. Das, M. van Gorcum, H. Perrin, B. Andreotti, J. H. Snoeijer. Droplets move over viscoelastic substrates by surfing a ridge Nature Communications, 6, 7891 (2015)

[49] Yoon J, Cai S, Suo Z and Hayward R C. Poroelastic swelling kinetics of thin hydrogel layers: comparison of theory and experiment Soft Matter 6(23), 6004 (2010)

[50] S. Matsukawa, I Ando. A Study of Self-Diffusion of Molecules in Polymer Gel byPulsed-Gradient Spin-Echo ${ }^{1} \mathrm{H}$ NMR Macromolecules, 29, 7136-7140 (1996).

[51] Dalby, M. J., Riehle, M. O., Yarwood, S. J., Wilkinson, C. D. and Curtis, A. S. Nucleus alignment and cell signaling in fibroblasts: response to a micro-grooved topography. Exp. Cell Res. 284, 274 ?282 (2003).

[52] Diehl, K. A., Foley, J. D., Nealey, P. F. and Murphy, C. J. Nanoscale topography modulates corneal epithelial cell migration. J Biomed Mat Res Part A, 2005. 75A(3): 603-11.

[53] Kaiser, J. P., Reinmann, A. and Bruinink, A. The effect of topographic characteristics on cell migration velocity. Biomaterials 27, 5230 ?5241 (2006).

[54] Park J et al. Directed migration of cancer cells guided by the graded texture of the underlying matrix. Nat. Mat. 15(7) 792-801 (2016). 